\title{
A Novel MMC Control Scheme to Increase the DC Voltage in HVDC Transmission Systems
}

\author{
Rui $\mathrm{Li}^{\mathrm{a}, *}$, John E. Fletcher ${ }^{\mathrm{b}}$
}

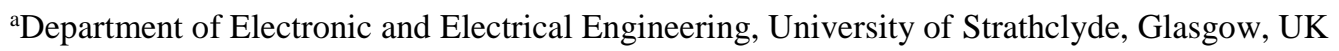 \\ ${ }^{\mathrm{b}}$ School of Electrical Engineering and Telecommunications, University of New South Wales, Sydney, Australia \\ E-mail: rui.li@strath.ac.uk
}

\begin{abstract}
This research investigates third harmonic injection applied to a modular multilevel converter (MMC) to generate a higher DC voltage. This is achieved using a proposed novel control scheme that activates existing submodules (SMs) in the converter arms. The technique is fundamentally different to, and does the reverse of, the well-known third harmonic injection techniques utilized to increase the AC output voltage in three-phase converter systems for a given DC link voltage. In the proposed scheme, the number of inserted SMs in each converter leg is greater than the number of SMs per arm, whence the MMC can operate with a higher DC link voltage while the SM number per arm and their capacitor voltages remain unchanged. This lowers the DC current and the DC transmission loss is significantly reduced by $22 \%$. Station conduction losses with the operational scheme are lowered by $2.4 \%$. The semiconductor current stresses are also lowered due to the reduced DC component of arm currents. Additionally, the phase energy variation is reduced by $18 \%$, which benefits circulating current control. The operating principles are presented in detail and mathematical models for conduction losses, energy variation, and circulating voltage are derived. Simulation of a point-to-point HVDC system demonstrates the effectiveness of the proposed MMC operational scheme.
\end{abstract}

Index Terms-DC transmission loss; HVDC transmission; modular multilevel converter (MMC); sinusoidal modulation; third harmonic injection. 


\section{INTRODUCTION}

Modular multilevel converter (MMC) deployment is increasing in HVDC application, due to its advantages over thyristor based line commutated converters (LCCs) and two-level voltage source converters (VSCs). MMC technology is more likely to be used to transmit renewable energy with large scale power capacity. A three-phase transformer is used on the MMC AC-side for converter isolation and to suit the required AC and DC voltages. Thus sinusoidal modulation can generate the required voltage and control the MMC. Additionally, it has good harmonic characteristics and can be simply implemented in digital controllers. As a result, sinusoidal modulation is widely used in the control of MMCs [1-5].

Selective harmonic elimination (SHE) modulation is used to control the MMC in [6, 7] to eliminate low order harmonics and reduce switching losses. Trapezoidal modulation has been proposed for the solid-state DC transformer and in the hybrid cascaded MMC, [8] and [9] respectively, to reduce switching losses and effectively utilize the DC voltage to produce an output AC voltage with higher fundamental amplitude. However, this is achieved at the expense of higher capital cost and a larger footprint.

By adding zero-sequence components into the reference voltage, discontinuous modulation is achieved which increases the modulation gain and reduces switching losses [10, 11]. However, additional submodules (SMs) are required in each arm to avoid over-modulation and reduce SM capacitor voltage ripple. In [12], a second harmonic is injected into the arm current to optimize the current distribution among the SMs and reduce the SM capacitor voltage ripple, but at the expense of higher semiconductor current stresses.

Third harmonic injection is another attractive approach for MMC control, as it improves the DC voltage utilization ratio. In [13], the MMC was first proposed for high voltage application and triplen harmonics, mainly third harmonic, were injected to effectively utilize the DC voltage. The advantage of third harmonic injection over sinusoidal modulation is discussed in [14] and the system performance under a DC fault is improved. However, the influence of the third harmonic during normal operation is not considered.

MMC dynamics under both balanced and unbalanced grid conditions with third harmonic injection are evaluated in [15]. However, the influence of the third harmonic on losses and SM capacitance is not considered. Modified space vector (SV) nearest level modulation (NLM) is discussed in [16] to increase the DC voltage utilization ratio thus reduce the DC voltage. However, the reduced DC voltage means a higher DC current in order to transfer rated power, resulting in higher power transmission losses. 
This paper is a post-print of a paper submitted to and accepted for publication in Electric Power Systems Research and is subject to ELSEVIER Copyright. The copy of record is available at ELSEVIER Digital Library.

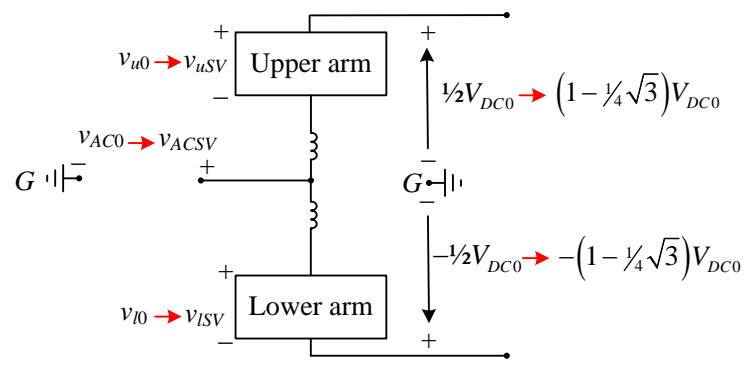

Fig. 1. The MMC circuit for one phase, where the voltage measurements of the sinusoidal modulation and the proposed control are illustrated before and after the arrows respectively.

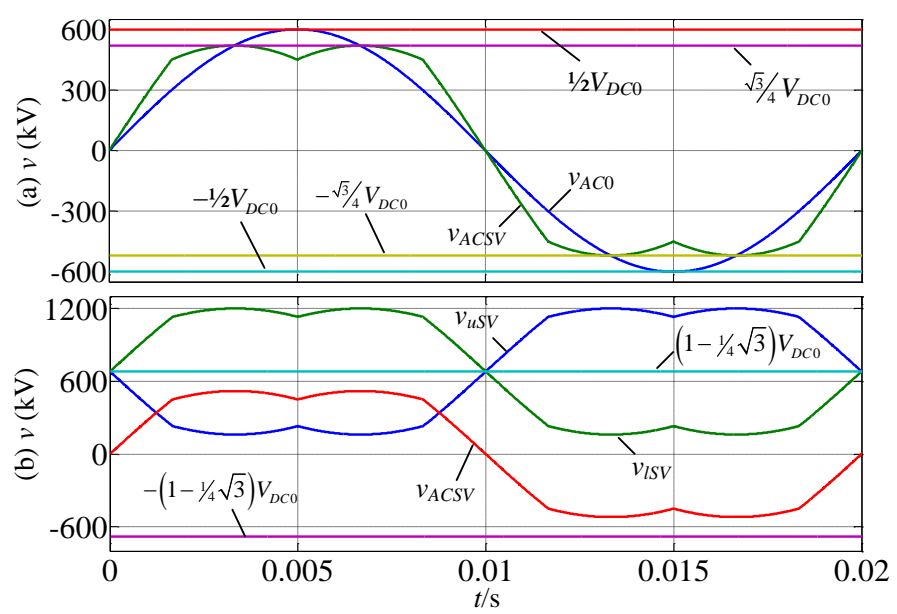

Fig. 2. Reference voltages to generate the fundamental voltage amplitude $\frac{1}{2} V_{D C 0}$ : (a) comparison between sinusoidal modulation and third harmonic injection, where $V_{D C 0}=1200 \mathrm{kV}$, and (b) proposed higher DC voltage operation, where $V_{D C S V}=(2-1 / 2 \sqrt{3}) V_{D C 0}=1361 \mathrm{kV}$.

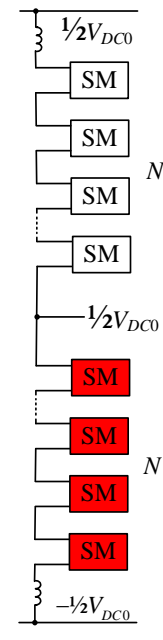

(a)

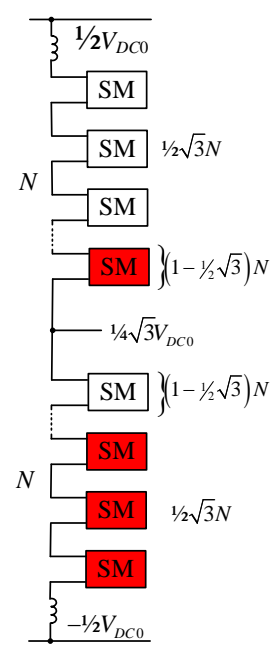

(b)

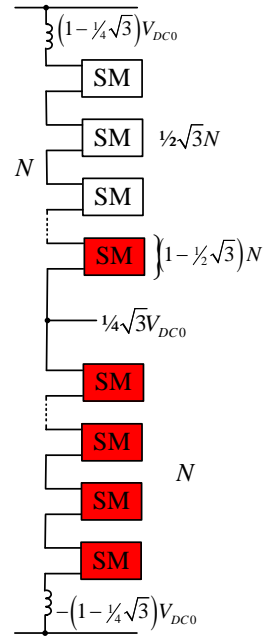

(c)

Fig. 3. Bypassed and inserted SMs per leg to generate the fundamental voltage amplitude $1 / 2 V_{D C 0}$, where the inserted SMs are shaded and $1 / 2 \sqrt{3} N$ is assumed to be an integer: (a) sinusoidal modulation , (b) conventional third harmonic injection, and (c) proposed higher DC voltage operation.

Although third harmonic injection is extensively used in MMC control, its use in increasing the DC voltage has not been fully explored in the MMC [13-16]. By injecting the third harmonic, the DC voltage utilization ratio is increased 
from $1 / 2$ to $1 / \sqrt{3}[10,17]$. The MMC circuit for one phase is shown in Fig. 1, where the voltage measurements of the sinusoidal modulation and the proposed control are illustrated before and after the arrows respectively. Compared with sinusoidal modulation, the maximum output voltage peak with reference to the mid-point of the DC link is reduced from $1 / 2 V_{D C 0}$ to $1 / 4 \sqrt{3} V_{D C 0}$ as demonstrated in Fig. 2 (a) and Fig. 3 (b), while the amplitude of the output fundamental voltage remains $1 / 2 V_{D C 0}$, where $V_{D C 0}$ is the DC voltage. For simplicity, it is assumed that $1 / 2 \sqrt{3} N$ is an integer, where $N$ is the SM number per arm. Only $1 / 2 \sqrt{3} N$ of the SMs are utilized when third harmonic injection is used rather than sinusoidal modulation. In real application, the potentially used SM number is $\operatorname{ceil}(1 / 2 \sqrt{3} N)$, which is close to $1 / 2 \sqrt{3} N$ given that HVDC systems typically use hundreds of SMs per arm. To utilize the remaining $13.4 \%$ SMs, a novel control scheme for MMCs is proposed that results in a higher DC voltage. This principle reduces the DC current, yielding lower overall system losses.

This study focuses on the novel higher DC voltage operation of MMC incorporating third harmonic injection, in a HVDC system. The paper is organized as follows. In Section II, the principles to operate a MMC with higher DC voltage are discussed along with resultant SM capacitance requirements, power losses, and circulating voltage. In Section III, the control strategy is presented. System performance using the proposed MMC operational mode is assessed in Section IV, by considering a point-to-point HVDC link. Section V draws the conclusions.

\section{Higher DC VOLTAGE Operation}

An MMC conventionally operates with the requirement that the number of inserted/bypassed SMs in each converter leg equals the SM number per arm $N[13,18]$. In this section, a novel MMC control scheme is proposed, where the inserted SM number per leg is greater than $N$. The technique, whilst achieving a higher DC voltage hence reduced DC current and associated losses, also has a significant positive impact on other performances of the station.

\section{A. Operating Principle}

Conventionally, third harmonic injection is used to extend the linear operating range of an MMC when compared with sinusoidal modulation. With AC-side voltage and DC voltage remaining unchanged, the MMC can effectively utilize the DC voltage and generate relatively higher AC line voltage under abnormal conditions, e.g. system-level events causing raised AC voltage or reduced DC voltage, etc., benefitting from the higher DC voltage utilization ratio provided by third harmonic injection [14-16]. Conventionally, in each arm, the number of inserted SMs equals the number of bypassed SMs: 


$$
\left\{\begin{array}{l}
N_{l u 0}+N_{I l 0}=N \\
N_{B u 0}+N_{B l 0}=N
\end{array}\right.
$$

where $N_{I u 0}$ and $N_{I I 0}$ are the numbers of inserted SMs in the upper and lower arms respectively; $N_{B u 0}$ and $N_{B l 0}$ are the numbers of bypassed SMs in the upper and lower arms respectively, under conventional control.

As shown in Fig. 3 (b), only $1 / 2 \sqrt{3} N$ SMs are utilized per arm in conventional third harmonic injection. If the other redundant $(1-1 / 2 \sqrt{3}) N$ SMs were removed from the MMC, it would increase the SM capacitor voltages and result in high voltage stresses on semiconductors and SM capacitors. Alternatively, with the SM capacitor voltages unchanged, the $(1-1 / 2 \sqrt{3}) N$ redundant SMs can be used to support a higher DC voltage, Fig. 3 (c). This forms the basis of the proposed novel control scheme. With this novel higher DC voltage operation, MMC operation does not comply with the requirement that the inserted SM number per leg equals the SM number per arm $N$, as depicted by (1). Now, in each converter leg, the inserted SMs are always more than the bypassed SMs to support the higher DC voltage while the SM capacitor voltages remain unchanged:

$$
\left\{\begin{array}{l}
N_{\text {LUSV }}+N_{I I S V}=(2-1 / 2 \sqrt{3}) N>N \\
N_{B U S V}+N_{B I S V}=1 / 2 \sqrt{3} N<N
\end{array}\right.
$$

where $N_{I L S V}$ and $N_{I I S V}$ are the numbers of inserted SMs in the upper and lower arms respectively and $N_{B u S V}$ and $N_{B I S V}$ are the numbers of bypassed SMs in the upper and lower arms respectively, in the proposed scheme.

Equation (2) shows the ideal numbers of the inserted and bypassed SMs, in order to simplify analysis. In real application, the SM number must be integers and are govern by:

$$
\left\{\begin{array}{l}
N_{\text {LLSV }}+N_{I I S V}=\text { floor }[(2-1 / 2 \sqrt{3}) N] \\
N_{B U S V}+N_{B I S V}=2 N-\text { floor }[(2-1 / 2 \sqrt{3}) N]
\end{array}\right.
$$

In this new MMC control scheme, the inserted SMs in each converter leg are greater than the bypassed SMs. Thus the MMC operates with a higher DC link voltage while the SM number per arm and the SM capacitor voltage remain unchanged. As demonstrated in Fig. 2 (b) and Fig. 3 (c), the maximum and minimum inserted or bypassed SM numbers per arm are

$$
\begin{gathered}
\left\{\begin{array}{l}
N_{l u \max S V}=N_{I l \max S V}=N \\
N_{B u \max S V}=N_{B l \max S V}=1 / 2 \sqrt{3} N
\end{array}\right. \\
\left\{\begin{array}{l}
N_{l u \min S V}=N_{I l \min S V}=(1-1 / 2 \sqrt{3}) N \\
N_{B u \min S V}=N_{B l \min S V}=0
\end{array}\right.
\end{gathered}
$$


In the proposed control scheme, the DC voltage is thus increased to:

$$
V_{D C S V}=[N+(1-1 / 2 \sqrt{3}) N] V_{D C 0} / N=(2-1 / 2 \sqrt{3}) V_{D C 0}=113.4 \% V_{D C 0}
$$

The DC voltage is increased by $13.4 \%$ while the SM number per arm and SM capacitor voltages are unchanged. Because of the increased DC voltage, the galvanic isolation requirements of the MMC station and DC cable are expected to increase by $13.4 \%$. This may increase the capital cost of the station and the DC cables. However, the DC current $I_{D C S V}$ is reduced to $88.2 \%$ to transfer the rated power and the DC transmission losses $P_{\text {loss } D C S V}$ is lowered by $22.2 \%$ on the assumption that the DC cable resistance remains unchanged. As the arm current is the sum of half the AC current and a third of the DC current, the arm current is thus lowered, resulting in reduced current stresses in the switching devices and lower station converter losses.

The penalty of the novel control scheme is the constraint of the AC voltage output capability, thereby limiting the linear operating range. As shown in Fig. 3 (c), the maximum output voltage peak when utilising the proposed technique is:

$$
V_{\max S V}=1 / 2 V_{D C S V}-(1-1 / 2 \sqrt{3}) V_{D C 0}=1 / 4 \sqrt{3} V_{D C 0}
$$

Although the DC voltage $V_{D C S V}$ is increased to $(2-1 / 2 \sqrt{3}) V_{D C 0}$ using the proposed higher DC voltage operation, the maximum output voltage peak is lower than that with conventional control $V_{\max 0}$ :

$$
V_{\max S V}=1 / 2 \sqrt{3} V_{\max 0}<V_{\max 0}=1 / 2 V_{D C 0} .
$$

Compared to conventional third harmonic injection, Fig. 3 (b), the linear operating range of an MMC with the proposed scheme is reduced under abnormal conditions, e.g. system-level events causing raised AC voltage or reduced DC voltage, etc. This also has negative influence on the active and reactive power output range on the MMC AC-side in abnormal conditions. However, under normal operation, the MMC fundamental output voltage is the same as that with conventional control $v_{A C 0}$, benefiting from the injected third harmonic:

$$
v_{A C S V}=v_{A C 0}+1 / 2 m_{h S V} V_{D C 0} \sin 3 \omega t=1 / 2 V_{D C 0}\left(m_{0} \sin \omega t+m_{h S V} \sin 3 \omega t\right)
$$

where $\omega$ is the grid angular frequency; $m_{0}$ is the modulation index with sinusoidal modulation; and $m_{h S V}$ is the modulation index of third harmonic. Based on (6), $v_{A C S V}$ is rewritten as

$$
v_{A C S V}=m_{S V} \frac{V_{D C S V}}{\sqrt{3}} \sin \omega t+1 / 2 m_{h S V} V_{D C 0} \sin 3 \omega t=1 / 6(4 \sqrt{3}-3) m_{S V} V_{D C 0} \sin \omega t+1 / 2 m_{h S V} V_{D C 0} \sin 3 \omega t .
$$


Comparing (10) with (9), the modulation index in the proposed scheme, $m_{S V}$, is:

$$
m_{S V}=\frac{1 / 2 m_{0}}{1 / 6(4 \sqrt{3}-3)}=\frac{3+4 \sqrt{3}}{13} m_{0}
$$

\section{B. Station Conduction Loss Comparisons}

As the arm currents of the MMC have to flow through hundreds of semiconductors in HVDC applications, the conduction losses are higher than the switching losses and are the dominant part of station converter losses [19-21]. Thus, the influence of the proposed control on the conduction losses are detailed in this section.

As the transferred power is assumed fixed, the AC current with the proposed higher DC voltage operation, $i_{A C S V}$, remains the same as with conventional control $i_{A C 0}$ and can be expressed in terms of the DC current $I_{D C 0}$ [22], considering the DC and fundamental frequency components:

$$
i_{A C S V}=i_{A C 0}=I_{m 0} \sin (\omega t-\varphi)=\frac{4 I_{D C 0}}{3 m_{0} \cos \varphi} \sin (\omega t-\varphi)
$$

where $I_{m 0}$ is the AC current amplitude and $\varphi$ is the phase angle between the phase voltage and current. Considering the DC and fundamental frequency components, the arm currents are

$$
\begin{gathered}
i_{u S V}=1 / 2 i_{A C S V}+1 / 3 I_{D C S V}=1 / 3 I_{D C 0}\left[\frac{2 \sin (\omega t-\varphi)}{m_{0} \cos \varphi}+\frac{8+2 \sqrt{3}}{13}\right] \\
i_{I S V}=-1 / 2 i_{A C S V}+1 / 3 I_{D C S V}=1 / 3 I_{D C 0}\left[-\frac{2 \sin (\omega t-\varphi)}{m_{0} \cos \varphi}+\frac{8+2 \sqrt{3}}{13}\right] .
\end{gathered}
$$

Although the DC voltage is increased with the proposed operation, the semiconductor number in the current path remains unchanged, $N$. For simplicity, the forward voltages of the IGBT and anti-parallel diode are assumed to be identical and denoted as $V_{f d}$. Thus, from (13), the conduction losses of the converter when utilising higher DC voltage operation is

$$
P_{\text {loss } V}=\frac{6}{2 \pi} \int_{0}^{2 \pi} N V_{f d}\left|i_{u S V}\right| d(\omega t)=\frac{4 N V_{f d} I_{D C 0}}{\pi}\left[\frac{2 \sqrt{169-(19+8 \sqrt{3}) m_{0}^{2} \cos ^{2} \varphi}}{13 m_{0} \cos \varphi}+\frac{8+2 \sqrt{3}}{13} \arccos \frac{\sqrt{169-(19+8 \sqrt{3}) m_{0}^{2} \cos ^{2} \varphi}}{13}\right]
$$

As the circulating current can be controlled around zero by proportional-resonant (PR) control, its influence on conduction losses are slight, and not accounted for in (15).

A station converter with parameters listed in Table I is tested, where $N$ is chosen as 24 with half-bridge (HB) SMs operating at $50 \mathrm{kV}$, to reduce model complexity. In a typical application, hundreds of submodules with voltage rating of 
several kilovolts are required. From (15), the conduction power losses of the test station with conventional control and the proposed operational mode are: 10.87 MW and 10.61 MW, respectively. The station conduction losses of the proposed operation are reduced by $2.4 \%$.

\section{SM Capacitor Voltage Balancing}

The specified maximum capacitor voltage ripple generally determines the SM capacitance requirement. This section presents the process of sizing the capacitances and the SM voltage balancing approach for the proposed MMC operation. According to (6) and (9), the upper and lower arm voltages can be expressed as

$$
\begin{aligned}
& v_{u S V}=1 / 2 V_{D C 0}\left[2-1 / 2 \sqrt{3}-m_{0} \sin \omega t-m_{h S V} \sin 3 \omega t\right] \\
& v_{I S V}=1 / 2 V_{D C 0}\left[2-1 / 2 \sqrt{3}+m_{0} \sin \omega t+m_{h S V} \sin 3 \omega t\right] .
\end{aligned}
$$

The injected third harmonic only exists in the converter phase voltages referenced to the mid-point of the DC link and does not affect the qualities of the line voltages and currents.

TABLE I

\begin{tabular}{|c|c|c|}
\hline PARAMETER & CONVENTIONAL CONTROL & PROPOSED HIGHER DC VOLTAGE OPERATION \\
\hline AC grid voltage & \multicolumn{2}{|c|}{$480 \mathrm{kV}$} \\
\hline Power rating & \multicolumn{2}{|c|}{$1650 \mathrm{MW}$} \\
\hline Arm inductance & \multicolumn{2}{|c|}{$0.05 \mathrm{pu}$} \\
\hline Number of SMs per arm & \multicolumn{2}{|c|}{24} \\
\hline SM capacitance & \multicolumn{2}{|c|}{$334 \mu \mathrm{F}$} \\
\hline SM capacitor voltage & \multicolumn{2}{|c|}{$50 \mathrm{kV}$} \\
\hline DC voltage & $1200 \mathrm{kV}$ & $1350 \mathrm{kV}$ \\
\hline DC current & $1375 \mathrm{~A}$ & $1222 \mathrm{~A}$ \\
\hline Arm current peak & $1604 \mathrm{~A}$ & $1553 \mathrm{~A}$ \\
\hline Station conduction loss & $P_{l o s s 0}$ & $0.976 P_{\text {loss } 0}$ \\
\hline DC transmission loss & $P_{\text {loss DC0 }}$ & $0.778 P_{l o s s D C 0}$ \\
\hline
\end{tabular}

Comparison between Conventional Control and the Proposed Technique

Integrating the product of the arm current and voltage, as depicted by (13), (14), (16), and (17), the upper and lower arm energy variations with the proposed higher DC voltage operation are

$$
\begin{aligned}
\Delta E_{u S V}(\omega t)=\frac{1}{\omega} \int_{0}^{\omega t} v_{u S V} i_{u S V} d(\omega t)= & \frac{V_{D C 0} I_{D C 0}}{6 \omega}\left\{\frac{8+2 \sqrt{3}}{13} m_{0}[\cos (\omega t)-1]-\frac{4-\sqrt{3}}{m_{0} \cos \varphi}[\cos (\omega t-\varphi)-\cos \varphi]+\frac{1}{2 \cos \varphi}[\sin (2 \omega t-\varphi)+\sin \varphi]\right\} \\
& -\frac{m_{h S V} V_{D C 0} I_{D C 0}}{6 \omega}\left\{\frac{1}{m_{0} \cos \varphi}[1 / 2 \sin (2 \omega t+\varphi)-1 / 4 \sin (4 \omega t-\varphi)-3 / 4 \sin \varphi]-\frac{8+2 \sqrt{3}}{39}[\cos (3 \omega t)-1]\right\}
\end{aligned}
$$




$$
\begin{aligned}
\Delta E_{I S V}(\omega t)=\frac{1}{\omega} \int_{0}^{\omega t} v_{I S V} i_{I S V} d(\omega t)= & \frac{V_{D C 0} I_{D C 0}}{6 \omega}\left\{-\frac{8+2 \sqrt{3}}{13} m_{0}[\cos (\omega t)-1]+\frac{4-\sqrt{3}}{m_{0} \cos \varphi}[\cos (\omega t-\varphi)-\cos \varphi]+\frac{1}{2 \cos \varphi}[\sin (2 \omega t-\varphi)+\sin \varphi]\right\} \\
& +\frac{m_{h S V} V_{D C 0} I_{D C 0}}{6 \omega}\left\{-\frac{1}{m_{0} \cos \varphi}[1 / 2 \sin (2 \omega t+\varphi)-1 / 4 \sin (4 \omega t-\varphi)-3 / 4 \sin \varphi]-\frac{8+2 \sqrt{3}}{39}[\cos (3 \omega t)-1]\right\} .
\end{aligned}
$$

With $\pm 10 \%$ voltage ripple requirement [23], the SM capacitance with higher DC voltage operation can be derived based on the arm energy variation as depicted by (18) and (19).

In (13) and (14), the AC component peak of the arm current is greater than the DC component:

$$
\frac{2 I_{D C 0} \sin (\omega t-\varphi)}{3 m_{0} \cos \varphi}>\frac{8+2 \sqrt{3}}{39} I_{D C 0}
$$

As a result, the arm currents are both positive and negative within each period, which ensures sufficient charging and discharging capability for submodule capacitors in the proposed higher DC voltage operation [24].

According to the required arm reference voltages, as illustrated by (16) and (17), the inserted and bypassed SM numbers per arm are determined by the nearest level modulation (NLM) and are governed by (3). Then the SM capacitor voltage balancing control proposed in [25] is implemented in each arm and all the $N$ submodules per arm can be balanced, although the number of inserted and bypassed SMs is different to conventional MMC control.

\section{Circulating Voltages}

Assuming SM capacitor voltages in each arm remain balanced, the arm stored energy is

$$
E_{\text {arm }}=1 / 2 N C_{S M}\left(\frac{V_{D C 0}}{N}+\Delta v_{S M}\right)^{2}
$$

where $C_{S M}$ is the SM capacitance and $\Delta v_{S M}$ is the SM capacitor voltage ripple. Based on (21), the arm energy variation can be approximated as

$$
\Delta E_{a r m}=N C_{S M}\left(\frac{V_{D C 0}}{N}+\Delta v_{S M}\right) \Delta v_{S M} \approx C_{S M} V_{D C 0} \Delta v_{S M}
$$

Then the SM capacitor voltage ripple can be expressed by the arm energy variation:

$$
\Delta v_{S M}=\frac{\Delta E_{a r m}}{C_{S M} V_{D C 0}}
$$

According to (16), (17) and (23) and considering SM capacitor voltage ripple, the upper and lower arm voltages are:

$$
v_{u S V}=1 / 2\left[2-1 / 2 \sqrt{3}-m_{0} \sin \omega t-m_{h S V} \sin 3 \omega t\right]\left(V_{D C 0}+\frac{N \Delta E_{u S V}}{C_{S M} V_{D C 0}}\right)
$$




$$
v_{I S V}=1 / 2\left[2-1 / 2 \sqrt{3}+m_{0} \sin \omega t+m_{h S V} \sin 3 \omega t\right]\left(V_{D C 0}+\frac{N \Delta E_{I S V}}{C_{S M} V_{D C 0}}\right)
$$

Then the sum of the upper and lower arm voltages is

$$
\begin{aligned}
& v_{u S V}+v_{L S V}=(2-1 / 2 \sqrt{3}) V_{D C 0}+v_{\text {cirs } S V} \\
& +\frac{(4+\sqrt{3}) N I_{D C 0}\left(m_{S V} \sin \omega t+m_{h S V} \sin 3 \omega t\right)}{39 \omega C_{S M}}\left\{-\frac{m_{h S V}}{3}[\cos (3 \omega t)-1]+\frac{338}{(8+2 \sqrt{3})^{2} m_{0} \cos \varphi}[\cos (\omega t-\varphi)-\cos \varphi]-m_{0}[\cos (\omega t)-1]\right\}
\end{aligned}
$$

where $v_{c i r S v}$ is the dominant circulating voltage, specifically

$$
v_{c i r S V}=\frac{(1-1 / 4 \sqrt{3}) N}{C_{S M} V_{D C 0}}\left(\Delta E_{I S V}+\Delta E_{u S V}\right)=\frac{(1-1 / 4 \sqrt{3}) N}{C_{S M} V_{D C 0}} \Delta E_{p S V}
$$

According to (18) and (19), the phase energy variation considering the third harmonic is

$$
\Delta E_{p S V}=\Delta E_{u S V}+\Delta E_{l S V}=\frac{V_{D C 0} I_{D C 0}}{6 \omega \cos \varphi}[\sin (2 \omega t-\varphi)+\sin \varphi]-\frac{m_{h S V} V_{D C 0} I_{D C 0}}{3 m_{0} \omega \cos \varphi}[1 / 2 \sin (2 \omega t+\varphi)-1 / 4 \sin (4 \omega t-\varphi)-3 / 4 \sin \varphi]
$$

The circulating current is generated by the circulating voltage, which is imposed on the upper and lower arm inductors and is proportional to the phase energy variation $\Delta E_{p S V}$, depicted by (27). As show in Fig. 4, the phase energy variation is reduced from $1.75 \mathrm{MJ}$ to $1.43 \mathrm{MJ}$ (by 18.3\%) using the proposed operation. This correspondingly reduces the circulating voltage and thereby benefits circulating current control.

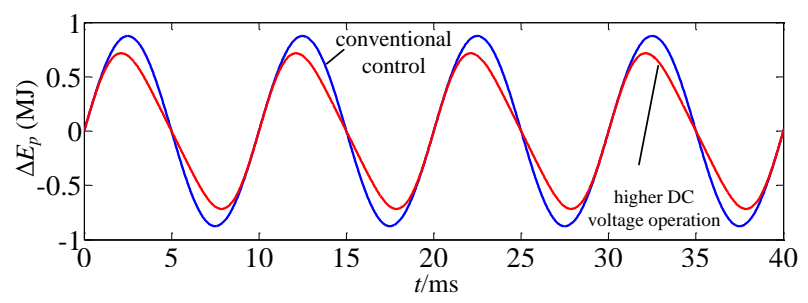

Fig. 4. Comparison of phase energy variation between conventional control and higher DC voltage operation, for $m_{0}=0.8, V_{D C 0}=1200 \mathrm{kV}, P=1650$ MW and $\varphi=0$.

Besides the dominant second component, the fourth component is introduced in the phase energy variation $\Delta E_{p S V}$, due to the injected third harmonic. The phase energy variation is independent of the modulation index and the third harmonic contributes to the reduction of the phase energy variation.

\section{CONTROL STRATEGY}

The control strategy of the proposed higher DC voltage operation is shown in Fig. 5. In DC voltage control mode, the DC voltage reference for higher DC voltage operation is set as: 


$$
V_{\text {DCSVref }}=V_{S M} \text { floor }[(2-1 / 2 \sqrt{3}) N]
$$

where $V_{S M}$ is the submodule capacitor voltage. Then the error between the reference $V_{D C S V r e f}$ and the feedback $v_{D C S V}$ is regulated by a PI controller, which sets the $d$-axis current reference $i_{d r e f}$, as shown in Fig. 5 . If the MMC was assigned to control active power, $i_{d r e f}$ would be set by the active power controller with the reference of $P_{r e f}$ and the actual power $P$ which is obtained by multiplying the $d$-axis voltage and current ( $v_{d}$ and $i_{d}$, Fig. 5). The $q$-axis current reference $i_{q r e f}$ can be similarly derived from the reactive power controller.

The $d$ - and $q$-axis currents $i_{d}$ and $i_{q}$ are obtained from the three-phase currents by a reference frame transformation and are as the feedback signals of the $d$ - and $q$-axis current loops. The outputs of the $d$ - and $q$-axis PI regulators are added to $\left(v_{d}-\omega L_{a r m} i_{q} / 2\right)$ and $\left(v_{q}+\omega L_{a r m} i_{d} / 2\right)$ respectively, where $L_{a r m}$ is the arm inductance. Thus the control between the $d$ - and $q$-axis is decoupled and the voltage references $e_{\text {dref }}$ and $e_{q r e f}$ are obtained [26].

Based on the reference voltages in $\alpha \beta$ coordinates ( $v_{\alpha r e f}$ and $v_{\beta r e f}$ ), a third harmonic injection block sets the reference voltages in $a b c$ coordinates ( $v_{\text {aref }}^{\prime}, v_{b r e f}^{\prime}$ and $v_{c r e f}^{\prime}$ ). Third harmonic is injected into the three phase sinusoidal voltages $v_{a}$, $v_{b}$, and $v_{c}$ by

$$
\left\{\begin{array}{l}
v_{a S V}=v_{a}-v_{h S V} \\
v_{b S V}=v_{b}-v_{h S V} \\
v_{c S V}=v_{c}-v_{h S V}
\end{array}\right.
$$

where $v_{a S V}, v_{b S V}$, and $v_{c S V}$ are the three phase voltages with third harmonic; and

$$
v_{h S V}=1 / 2\left[\max \left(v_{a}, v_{b}, v_{c}\right)+\min \left(v_{a}, v_{b}, v_{c}\right)\right] .
$$



Fig. 5. Control strategy of MMC with the proposed higher DC voltage operation. 
Besides the third harmonic, the voltage $v_{h S V}$ contains other triplen harmonics. However, the third harmonic is dominant in the triplen harmonics and the influence of other triplen harmonics $\left(h=9^{\text {th }}, 15^{\text {th }}, \ldots\right)$ is ignored (purely third harmonic injection is a valid concept for increasing the DC voltage utilization ratio by $15.5 \%$ ). The adopted third harmonic injection approach described by (30) and (31) has the same effect as SV modulation, as presented in [15, 17, 26]. Moreover, it is simple to implement and does not cause extra computational burden for the controller, even for an MMC with several hundred SMs per arm.

As shown in Fig. 5, a proportional-resonant (PR) control is used to suppress the circulating currents $i_{z a b c}$, which are obtained by filtering out the low frequency component of the sum of the upper and lower arm currents $\left(i_{u a b c}\right.$ and $i_{l a b c}$, Fig. 5). With the reference $i_{z a b c r e f}$ set at zero, the error between $i_{z a b c}$ and $i_{z a b c r e f}$ is regulated by the PR control and its output $\Delta v_{a b c r e f}$ is added to the three-phase references $v_{a b c r e f}^{\prime}$ to regulate the circulating currents.

\section{Performance EVAluation}

The performances of the proposed higher DC voltage operational mode are assessed using a point-to-point HVDC link model as shown in Fig. 6, in the MATLAB/Simulink ${ }^{\circledR}$ environment. The parameters of the two converters $\left(\mathrm{MMC}_{1}\right.$ and $\mathrm{MMC}_{2}$ ) are the same, as listed in Table $\mathrm{I}$. In the test system, $\mathrm{MMC}_{1}$ controls active power exchanged with grid $\mathrm{G}_{1}$ with unity power factor while $\mathrm{MMC}_{2}$ is configured to regulate the $\mathrm{DC}$ link voltage level at $\pm 600 \mathrm{kV}$, also at unity power factor. A conventional $\mathrm{Y} / \Delta$ transformer with grid-side earthed is used on the MMC AC-side to isolate the grid and the converter, thereby avoiding zero-sequence currents that would be induced in an earthed converter-side Y-connected winding using any third harmonic or triplen injection techniques.

The NLM is used in this paper to reduce the modulation complexity [25, 27]. The control frequency adopted is 40 $\mathrm{kHz}$, at which the voltages and currents are measured and the control signals are updated by the controller. With the SM capacitor voltage balancing control as presented in [25], the actual switching frequency of the IGBT in the MMC SMs is around $100 \mathrm{~Hz}$.

\section{A. Comparison between Conventional Control and Proposed Higher DC Voltage Operation}

According to (3), the inserted and bypassed SM numbers per leg with higher DC voltage operation are 27 and 21 respectively and the DC voltage is then calculated as $1350 \mathrm{kV}$, which is slightly lower than the ideal value of $1361 \mathrm{kV}$ obtained by (6). As mentioned, hundreds of SMs are usually required per arm in application, thus, the error between the ideal value and the available practical value is small. As shown in Fig. 7 (a), the DC voltage with conventional control is maintained at around $1200 \mathrm{kV}$ while with higher DC voltage operation it is increased to $1350 \mathrm{kV}$, benefiting from the higher DC voltage support capability of the proposed technique. This is achieved using the same number of SMs per 
$\operatorname{arm}(N=24)$ and SM capacitor voltage $(50 \mathrm{kV})$.

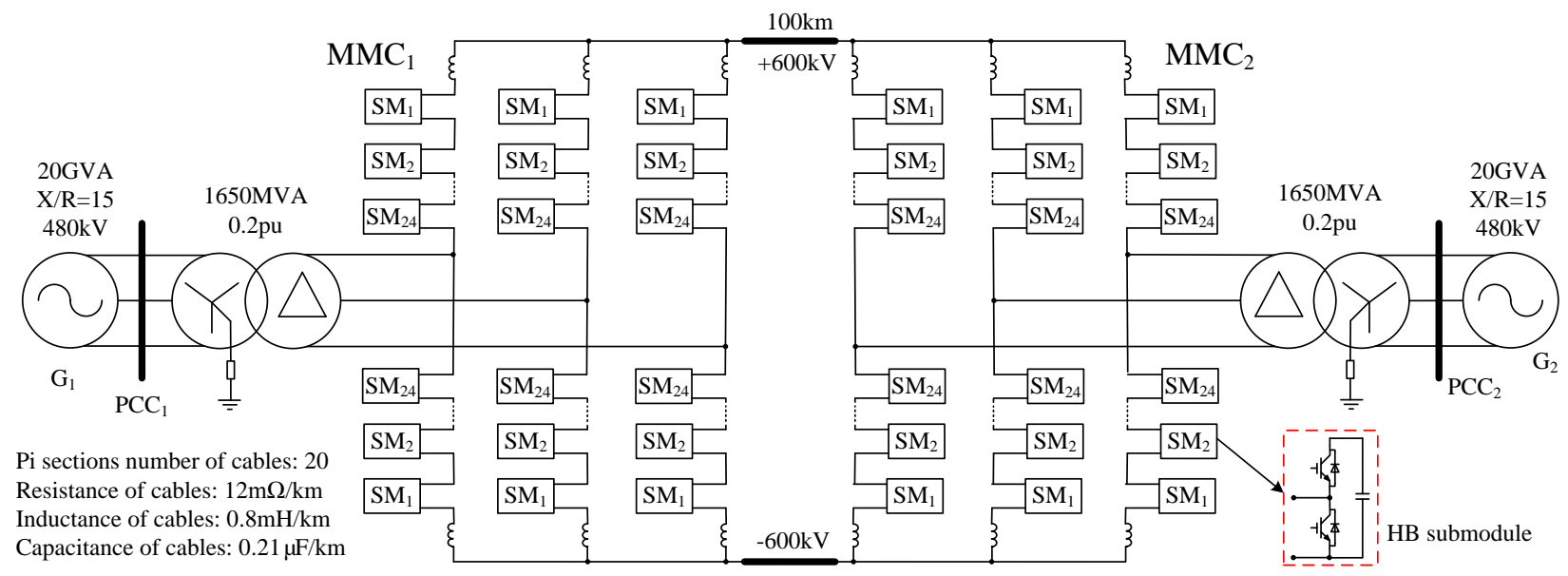

Fig. 6. Point-to-point HVDC link with half-bridge SM based MMCs where $N=24$.

The DC current waveforms are illustrated in Fig. 7 (b). The DC current with higher DC voltage operation is reduced to about $90 \%$ of that with conventional control, slightly higher than the ideal value $88.2 \%$. Correspondingly, the DC transmission loss is lowered to $80 \%$, in the simulation scenario. Due to the reduced DC current, the arm current peak with the proposed operation is reduced from $1.6 \mathrm{kA}$ to $1.5 \mathrm{kA}$ approximately, as shown in Fig. 7 (c), yielding lowered semiconductor current stresses.

The FFT analysis of the arm currents is shown in Fig. 8. Benefiting from the higher DC voltage generating capability, the DC component of the arm current in the proposed control is reduced from $458 \mathrm{~A}$ to $408 \mathrm{~A}$, yielding lower conduction losses. Additionally, the proposed method does not have any negative influence on the THD of the currents, which remains at around $2.4 \%$.

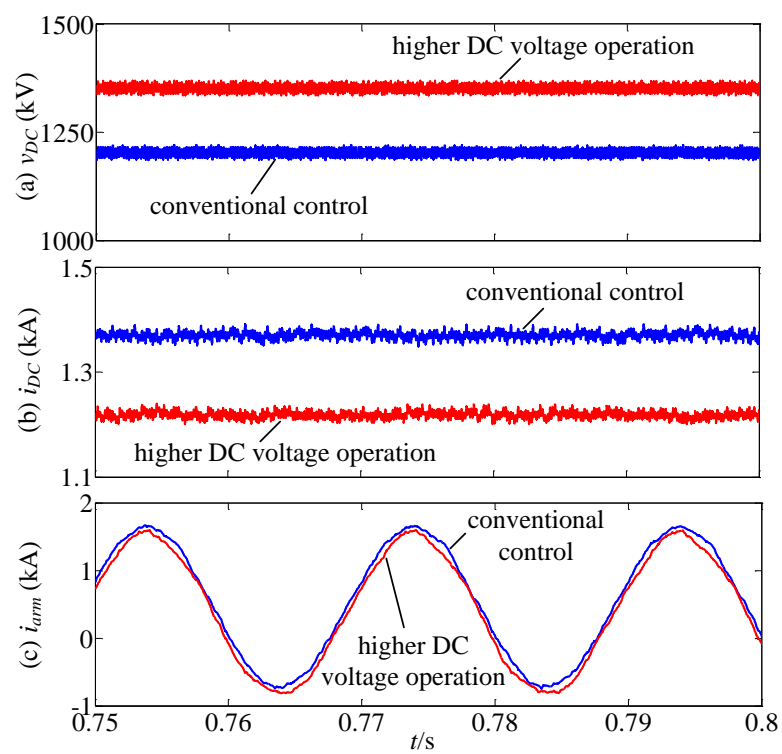

Fig. 7. Comparisons between conventional control and the proposed higher DC voltage operation: (a) DC voltages, (b) DC currents, and (c) arm currents. 


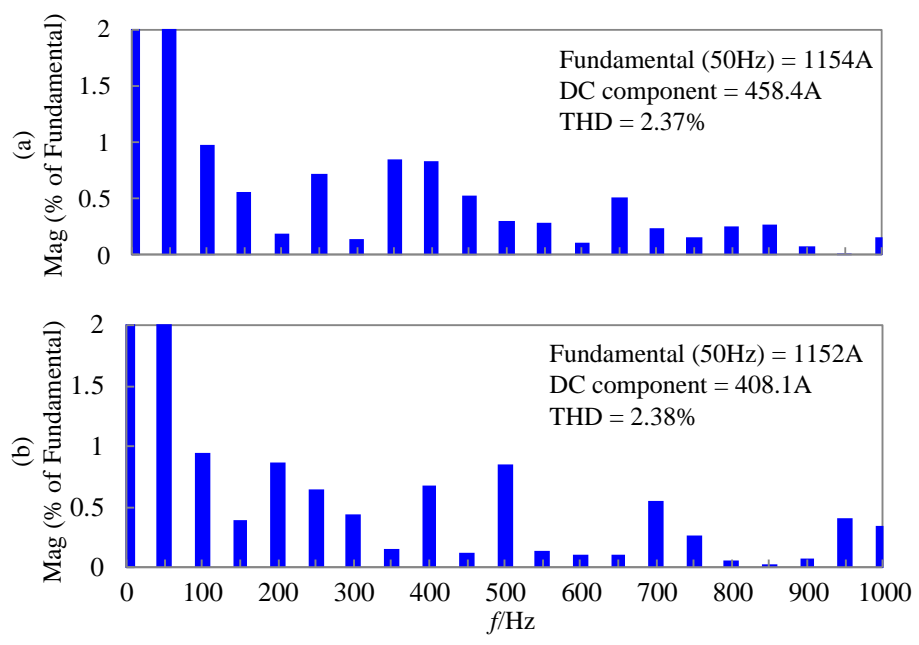

Fig. 8. FFT analysis of the arm currents with: (a) conventional control and (b) proposed higher DC voltage operation.

Table I summarizes the performances of the proposed operation. With the same SMs per arm (N=24), SM capacitor voltage $(50 \mathrm{kV})$, and transmitted power $(1650 \mathrm{MW})$, higher DC voltage operation reduces the DC current, thus the station conduction loss is reduced to $97.6 \%$. Additionally, the DC transmission loss in higher DC voltage operation is significantly reduced to $77.8 \%$. These improvements are achieved by simply altering the control scheme.

\section{B. Performance during Power Reversal}

To demonstrate the performance of the proposed control scheme during power reversal, $\mathrm{MMC}_{1}$ is initially commanded to import the rated power from grid $G_{1}$ to $G_{2}$ and the power flow direction is reversed at time $t=0.4 \mathrm{~s}$ to export the rated power from $\mathrm{G}_{2}$ to $\mathrm{G}_{1}$. The change rate of active power is restrained to $33 \mathrm{MW} / \mathrm{ms}$. In application, the power change rate is lower than the adopted value $(33 \mathrm{MW} / \mathrm{ms})$ and the system performance would be better than the presented results.

As shown in Fig. 9, minimal transient effects in the active and reactive powers and AC currents of $\mathrm{MMC}_{2}$ (converter station that regulates $\mathrm{DC}$ voltage level) are observed, as is also the case for $\mathrm{MMC}_{1}$ (converter that regulates active power). During active power reversal, as commanded by $\mathrm{MMC}_{1}$, the $\mathrm{DC}$ current reverses in all HVDC links, with limited overshoot. By using PR control, the circulating currents are controlled around zero and thus the arm currents of $\mathrm{MMC}_{1}$ and $\mathrm{MMC}_{2}$ are balanced, as shown in Fig. 9 (g) and (h). A limited drop in the DC link and SM capacitor voltages of both converter stations are observed, see Fig. 9 (f), (i), and (j). Additionally, all the SM capacitor voltages are balanced by the approach as presented in Section II C and the capacitor voltage ripple are limited in the range of $\pm 10 \%$. The sum of the SM capacitor voltages in the upper and lower arms are balanced benefitting from the fast response of the circulating current controller, see Fig. 9 (k) and (1).

Following power reversal, the HVDC transmission system returns quickly to its steady state operating point. The 
results in Fig. 9 show that an MMC with the proposed control scheme is able to operate over the entire operating range, with voltage and current stresses in the active and passive devices fully controlled.
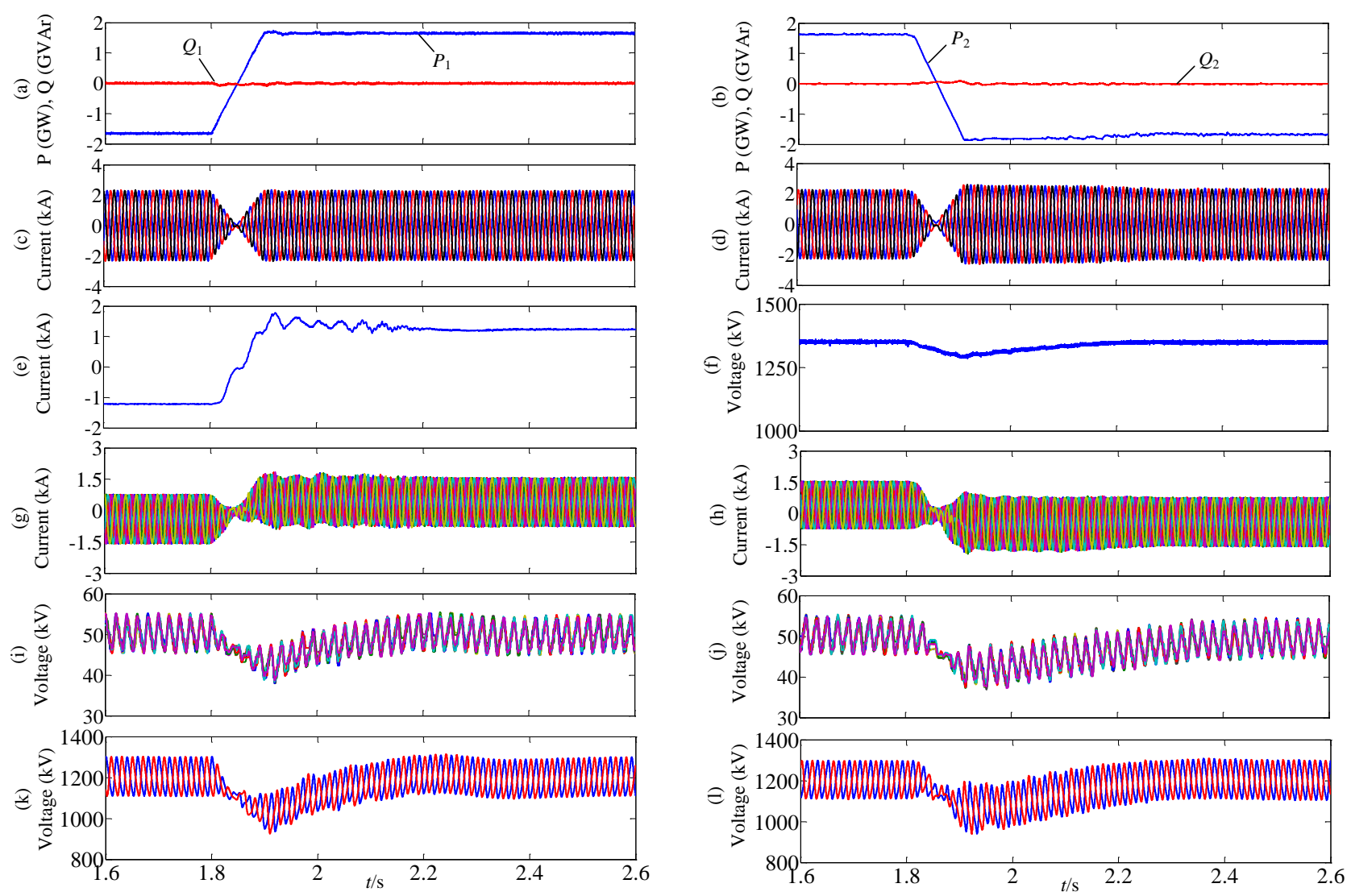

Fig. 9. Performance of $\mathrm{MMC}_{1}$ and $\mathrm{MMC}_{2}$ during power reversal: (a)-(b) active and reactive powers, (c)-(d) three-phase currents, (e) DC current, (f) DC voltage, (g)-(h) arm currents, (i)-(j) SM capacitor voltages in upper arm, and (k)-(l) the sum of SM capacitor voltages in upper and lower arms.

\section{DISCUSSION}

\section{A. Reduced SM Operation of the MMC with Unchanged DC Voltage}

The MMCs can operate with reduced SMs by using the proposed scheme, given that the DC voltage remains unchanged. Alternatively, with a certain SM number, the DC voltage of the MMC can be increased, e.g. for existing stations. These two MMC operational concepts both make use of the higher DC voltage utilization ratio of third harmonic injection. The former scheme is a straightforward alternative application of the proposed control.

The higher DC voltage operation capability is beneficial for the future power augmentation of existing HVDC systems, considering increased power demand or supply. For existing stations, the DC voltage increase is set according to the power augmentation demand and is flexible between 0 to $13.4 \%$, assuming the station and DC cable can tolerate the slightly increased DC voltage stress $(0 \sim 13.4 \%)$.

At the design stage, if the galvanic isolation of the station and DC cable is increased to meet the requirement with a 
higher DC voltage (13.4\%), the DC transmission loss, station conduction losses, and semiconductor current stresses can be lowered. Additionally, the transferred power can be increased by $13.4 \%$ by simply increasing the DC current to the original value. The higher DC voltage operation capability of the MMC makes the transferred power capacity more flexible, compared to that of the reduced SM operation.

In order to improve the MMC performance with fixed power demand and have power transfer headroom, the higher DC voltage operation of MMC HVDC system is considered in this paper, although the reduced SM operation is another viable option for the proposed control scheme.

\section{B. Zero-Sequence Current Consideration}

The earthing configuration of the interface transformer has a significant influence on the proposed control and modulation scheme. With the converter-side of the transformer earthed, zero-sequence current would flow to earth via the transformer neutral point when third or triplen series injection techniques are used, Fig. 10 (a), but this is an unusual

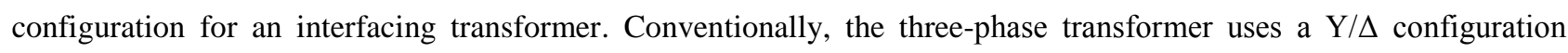
where the neutral is earthed on the Y-winding at the grid-side of the transformer [14, 15, 23], Fig. 10 (b) and Fig. 6. Although the third harmonic voltage is reflected on the virtual neutral voltage of the $\Delta$ winding at the converter-side of the transformer, the zero-sequence component of the three-phase currents is zero, as the Y/ $\Delta$ transformer arrangement in Fig. 10 (b) does not provide a zero-sequence current path. This makes the proposed control scheme viable for MMC HVDC systems with conventional $\mathrm{Y} / \Delta$ transformer earthed on the grid-side.

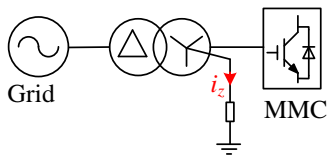

(a)

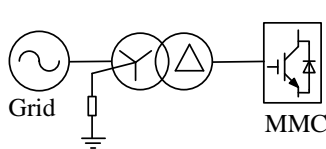

(b)

Fig. 10. Transformer configurations in MMC HVDC systems: (a) $\Delta / \mathrm{Y}$ transformer with converter-side earthed and (b) conventional $\mathrm{Y} / \Delta$ transformer with grid-side earthed.

\section{Switching Loss Consideration}

The switching losses are dependent on the method used to balance the submodule capacitor voltages. For example, a prior method [28] utilizes an average tolerance band and a sorting algorithm to balance the capacitor voltages. The technique results in a higher switching frequency and losses and therefore is not likely to be used in the HVDC application. With proper voltage balancing control, the switching frequency of the semiconductors in the MMC is low and can be reduced to $150 \mathrm{~Hz}$ or even less $[19,25]$. The third harmonic injection does necessarily increase the switching 
commutations at the voltage (current) peak. This has been clearly shown in Fig. 11, where both the proposed scheme and the sinusoidal modulation have an average switching frequency of around $100 \mathrm{~Hz}$.

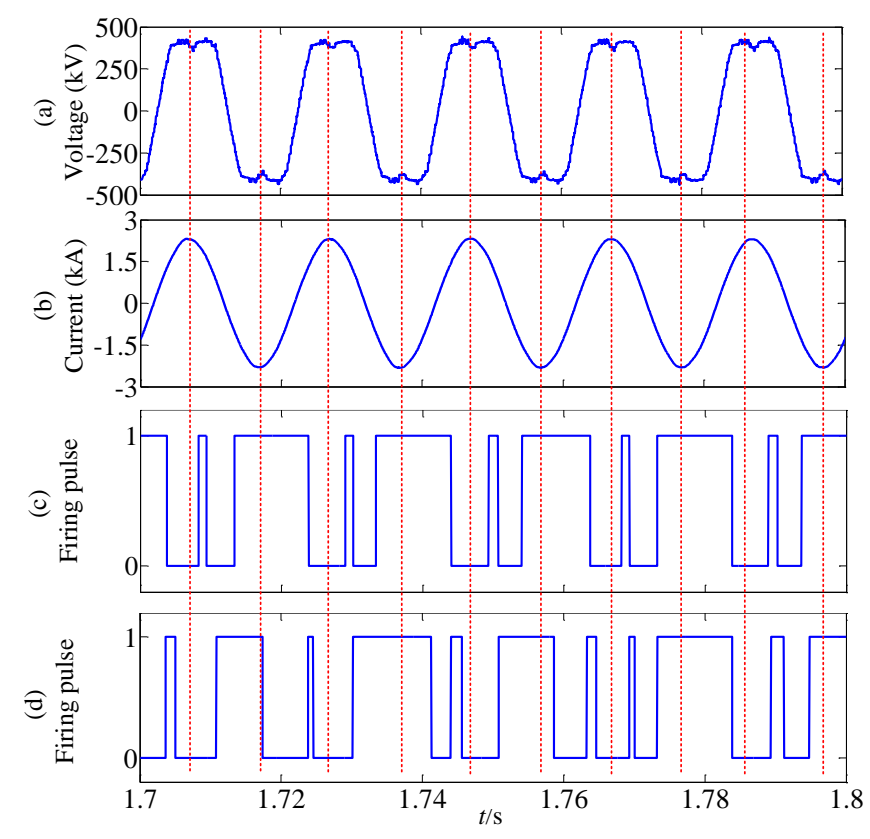

Fig. 11. Verification of the switching commutations at the voltage (current) peak: (a) output voltage with third harmonic injection, (b) output current with third harmonic injection, (c) firing pulse of the IGBT with third harmonic injection, and (d) firing pulse of the IGBT with sinusoidal modulation.

To further demonstrate the influence of the proposed control scheme on switching losses, the switching loss is evaluated based on the tested model with parameters listed in Table I. The switching losses are obtained according to the currents in the simulation and the switching characteristics of the semiconductor devices, as detailed in [28, 29]. The latter are derived from the datasheet provided by the manufacturer [30] and are stored in a lookup table. Assuming the semiconductors operate at the rated voltage of $2.5 \mathrm{kV}$ in the typical application, each semiconductor in the tested model with reduced SMs (24 SMs per arm) is equivalent to the series connection of 20 semiconductors in the typical application $(50 \mathrm{kV} / 2.5 \mathrm{kV}=20)$.

The switching losses of the proposed third harmonic injection are compared to that with sinusoidal modulation, Fig. 12. The switching losses increase with the power increasing from zero to the rated value and gradually reach the steady state. A close match is observed between sinusoidal and third harmonic injection techniques therefore the proposed control does not significantly influence the switching losses.

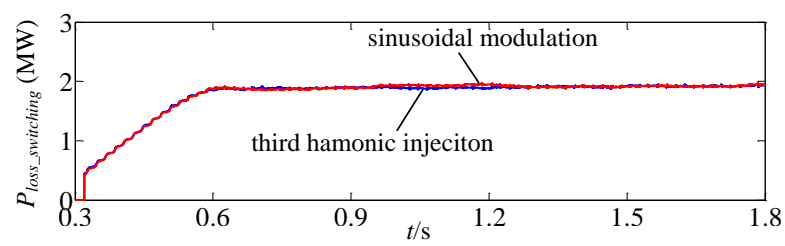

Fig. 12. Comparison of switching losses between sinusoidal modulation and third harmonic injection. 


\section{CONCLUSION}

A novel control scheme that results higher DC voltage operation of an MMC HVDC system incorporating third harmonic injection is proposed to better utilize converter SMs. In the proposed scheme, the inserted SM number in each converter leg is greater than the SM number per arm. Thus the DC voltage is increased by $13.4 \%$ while the SM number per arm and the SM capacitor voltage remain unchanged. Correspondingly, the DC current is lowered and the DC transmission loss is expected to be $77.8 \%$ of that with conventional control, for a given DC cable resistance. Additionally, station converter conduction losses with the proposed operation are reduced by $2.4 \%$. The semiconductor current stresses and phase energy variation are also reduced with the proposed control method. Simulation results were in good agreement with the analysis, which demonstrates the effectiveness of the novel higher DC voltage operation.

\section{REFERENCES}

[1] U. N. Gnanarathna, A. M. Gole, and R. P. Jayasinghe, "Efficient Modeling of Modular Multilevel HVDC Converters (MMC) on Electromagnetic Transient Simulation Programs," IEEE Trans. Power Del., vol. 26, pp. 316-324, 2011.

[2] G. P. Adam, O. Anaya-Lara, G. M. Burt, D. Telford, B. W. Williams, and J. R. McDonald, "Modular multilevel inverter: Pulse width modulation and capacitor balancing technique," IET. Power Electron., vol. 3, pp. 702-715, 2010.

[3] M. A. Perez, S. Bernet, J. Rodriguez, S. Kouro, and R. Lizana, "Circuit Topologies, Modeling, Control Schemes, and Applications of Modular Multilevel Converters," IEEE Trans. Power Electron., vol. 30, pp. 4-17, 2015.

[4] M. Hagiwara, K. Nishimura, and H. Akagi, "A Medium-Voltage Motor Drive With a Modular Multilevel PWM Inverter," IEEE Trans. Power Electron., vol. 25, pp. 1786-1799, 2010.

[5] Z. Ming, H. Long, Y. Wenxi, and L. Zhengyu, "Circulating Harmonic Current Elimination of a CPS-PWM-Based Modular Multilevel Converter With a Plug-In Repetitive Controller," IEEE Trans. Power Electron., vol. 29, pp. 2083-2097, 2014.

[6] G. Konstantinou, M. Ciobotaru, and V. Agelidis, "Selective harmonic elimination pulse-width modulation of modular multilevel converters," IET Power Electronics, vol. 6, pp. 96-107, 2013.

[7] G. S. Konstantinou, M. Ciobotaru, and V. G. Agelidis, "Operation of a modular multilevel converter with selective harmonic elimination PWM," in Power Electronics and ECCE Asia (ICPE \& ECCE), 2011 IEEE 8th International Conference on, 2011, pp. 999-1004.

[8] I. A. Gowaid, G. P. Adam, A. M. Massoud, S. Ahmed, D. Holliday, and B. W. Williams, "Quasi Two-Level Operation of Modular Multilevel Converter for Use in a High-Power DC Transformer With DC Fault Isolation Capability," IEEE Trans. Power Electron., vol. 30, pp. 108-123, 2015 .

[9] R. Li, G. Adam, D. Holliday, J. Fletcher, and B. Williams, "Hybrid Cascaded Modular Multilevel Converter with DC Fault Ride-Through Capability for HVDC Transmission System," IEEE Trans. Power Del., vol. PP, pp. 1-1, 2015.

[10]R. Picas, S. Ceballos, J. Pou, J. Zaragoza, G. Konstantinou, and V. G. Agelidis, "Improving capacitor voltage ripples and power losses of modular multilevel converters through discontinuous modulation," in Industrial Electronics Society, IECON 2013 - 39 th Annual Conference of the IEEE, 2013, pp. 6233-6238.

[11]R. Picas, S. Ceballos, J. Pou, J. Zaragoza, G. Konstantinou, and V. G. Agelidis, "Closed-Loop Discontinuous Modulation Technique for Capacitor Voltage Ripples and Switching Losses Reduction in Modular Multilevel Converters," IEEE Trans. Power Electron., vol. 30, pp. 4714$4725,2015$. 
This paper is a post-print of a paper submitted to and accepted for publication in Electric Power Systems Research and is subject to ELSEVIER Copyright. The copy of record is available at ELSEVIER Digital Library.

[12]A. Rasic, U. Krebs, H. Leu, and G. Herold, "Optimization of the modular multilevel converters performance using the second harmonic of the module current," in Power Electronics and Applications, 2009. EPE' 09. 13th European Conference on, 2009, pp. 1-10.

[13]A. Lesnicar and R. Marquardt, "An innovative modular multilevel converter topology suitable for a wide power range," in Power Tech Conference Proceedings, 2003 IEEE Bologna, 2003, p. 6 pp. Vol.3.

[14]G. Adam, R. Li, D. Holliday, S. Finney, L. Xu, B. Williams, et al., "Continued Operation of Multi-Terminal HVDC Networks Based on Modular Multilevel Converters," CIGRE, pp. 1-8, 2015.

[15]M. Saeedifard and R. Iravani, "Dynamic Performance of a Modular Multilevel Back-to-Back HVDC System," IEEE Trans. Power Del., vol. 25, pp. 2903-2912, 2010.

[16]K. H. Ahmed and G. P. Adam, "New modified staircase modulation and capacitor balancing strategy of 21-level modular multilevel converter for HVDC transmission systems," in Power Electronics, Machines and Drives (PEMD 2014), 7th IET International Conference on, 2014, pp. 1-6.

[17]F. Blaabjerg, A. Isidori, and F. M. Rossi, "Impact of modulation strategies on power devices loading for 10 MW multilevel wind power converter," in Power Electronics for Distributed Generation Systems (PEDG), 2012 3rd IEEE International Symposium on, 2012, pp. 751-758.

[18]T. Qingrui and X. Zheng, "Impact of Sampling Frequency on Harmonic Distortion for Modular Multilevel Converter," IEEE Trans. Power Del., vol. 26, pp. 298-306, 2011.

[19]Q. Tu, Z. Xu, and L. Xu, "Reduced Switching-Frequency Modulation and Circulating Current Suppression for Modular Multilevel Converters," IEEE Transactions on Power Delivery, vol. 26, pp. 2009-2017, 2011.

[20]S. Rohner, S. Bernet, M. Hiller, and R. Sommer, "Modulation, Losses, and Semiconductor Requirements of Modular Multilevel Converters," IEEE Transactions on Industrial Electronics, vol. 57, pp. 2633-2642, 2010.

[21]Z. Zhang, Z. Xu, and Y. Xue, "Valve Losses Evaluation Based on Piecewise Analytical Method for MMC\&\#x2013;HVDC Links," IEEE Transactions on Power Delivery, vol. 29, pp. 1354-1362, 2014.

[22]R. Li, J. E. Fletcher, L. Xu, D. Holliday, and B. W. Williams, "A Hybrid Modular Multilevel Converter with Novel Three-level Cells for DC Fault Blocking Capability," IEEE Trans. Power Del., vol. PP, pp. 1-1, 2015.

[23]J. Peralta, H. Saad, S. Dennetiere, J. Mahseredjian, and S. Nguefeu, "Detailed and Averaged Models for a 401-Level MMC-HVDC System," IEEE Trans. Power Del., vol. 27, pp. 1501-1508, 2012.

[24]R. Zeng, L. Xu, L. Yao, and B. W. Williams, "Design and Operation of a Hybrid Modular Multilevel Converter," IEEE Trans. Power Electron., vol. 30, pp. 1137-1146, 2015.

[25] Y. Feng, L. Weixing, W. Xitian, and X. Da, "Fast Voltage-Balancing Control and Fast Numerical Simulation Model for the Modular Multilevel Converter," IEEE Trans. Power Del., vol. 30, pp. 220-228, 2015.

[26]R. Li and D. G. Xu, "Parallel Operation of Full Power Converters in Permanent-Magnet Direct-Drive Wind Power Generation System," IEEE Trans. Ind. Electron., vol. 60, pp. 1619-1629, 2013.

[27]H. Saad, J. Peralta, S. Denneti, x00E, re, J. Mahseredjian, et al., "Dynamic Averaged and Simplified Models for MMC-Based HVDC Transmission Systems," IEEE Transactions on Power Delivery, vol. 28, pp. 1723-1730, 2013.

[28]A. Hassanpoor, S. Norrga, and A. Nami, "Loss evaluation for modular multilevel converters with different switching strategies," in 2015 9th International Conference on Power Electronics and ECCE Asia (ICPE-ECCE Asia), 2015, pp. 1558-1563.

[29]Q. Tu and Z. Xu, "Power losses evaluation for modular multilevel converter with junction temperature feedback," in Power and Energy Society General Meeting, 2011 IEEE, 2011, pp. 1-7.

[30]ABB, 5SNA 1200G450350 datasheet, Apr 2012. 